\section{Die Verdienste Rudolf Virchows um die Lehre von der Tuberkulose.}

Von Johannes Orth in Berlin.

Seit meiner Assistentenzeit habe ich in gewissen Tuberkulosefragen zu Virchows Lehre in Opposition gestanden; ich darf daher wohl annehmen, daß man mich nicht der Voreingenommenheit zeihen wird, wenn ich hier die Verdienste erörtern will, welche Virch ow sich um die Lehre von der Tuberkulose erworben hat. Es ist nicht das erstemal, daß ich in dieser Beziehung für ihn eintrete, sondern sowohl im ersten Bande meines Lehrbuches der speziellen pathologischen Anatomie (1883-1885) als auch seitdem bei verschiedenen Gelegenheiten habe ich die Virchowsche Lehre, soweit. sie nicht die Aetiologie und Begriffsbestimmung der jetzt allgemein als Tuberkulose bezeichneten Krankheit betraf, gegen Angriffe verteidigt. Es kann hier nicht meine Aufgabe sein, den Kampt in seine Einzel heitell zul verfolgen, vielmehr können nur in großen Zügen die Leistungen Virchows und ihre Bedeutung für den heutigen Stand der Lehre von der Tuberkulose dargelegt werden, während wegen mancher Einzelheiten auf meine erwähnten früheren Darlegungen verwiesell werden muß.

ln der Mitte des vorigen Jahrhunderts, als Rudolf Virchow schaffend in die Wissenschaft eingriff, herrschte in der Lehre von der Tuberkulose und der davon nicht scharf zu trennenden Skrofulose eine große Verwirrung, und fast jeder Pathologe hatte eine andere Meintung sowohl in bezug auf die Anatomie als auch in bezug auf die Abgrenzung der betreffenden Krankheit. Die Bezeichnung Tuberkel (vgl. meine Abhandlung: Zur Nomenklatur der Tuberkulose Sitzungsber. d. Preuß. Akad. d. Wiss. 1917 S.580) war aus einer allgemein deskriptiven allmählich eine spezifische geworden, d.h. man verstand unter Tuberkel nicht irgendein beliebiges knötchenförmiges Gebilde, solldern ein Gebilde eigener Art, über dessen Entstehung und Heranbildung allerdings die Ansichten weit auseinander gingen. B ayle war der Erste, der die Tuberkel in Verbindung mit einer eigenartigen (d'une nature speciale) Krankheit brachte, die er Dégénérescence oder Affection tuberculeuse nannte, für die dann Schönlein das Wort Tuberkulose prägte. Obwohl Bayle selbs für die Tuberkel das Wort Miliartuberkel eingeführt hatte, erklärte er doch nicht diesen für das pathognomonische Gebilde seiner Affection tuberculeuse, sondern die käsige Materie, welche vor allem bei der zu seiner Krankheit gehörigen Lungenerkrankung eine so groBe Rolle spielt. Schon damit war gesagt, daß nicht der Tuberkel das Erkennungszeichen der Tuberkulose sei, daB nicht nur da Tuberkulose sei, wo Tuberkel sich fänden, sondern daß der Befund von Käsemasse, die schließlich nichts anderes war als die früher sog. skrofulöse Materie, die Zugehörigkeit zur Tuberkulose bedinge. Das mußte um so mehr verwirrend wirken, als B ayle außer den verkäsenden Miliartuberkelı auch noch miliare Granulationen -- also schließlich doch auch miliare Knötchen, d.h. Miliartuberkel - anerkannte, die nicht verkästen und einer anderen Krankheit als der Tuberkulose alıgehören sollten. Die Lehre von den nicht verkäsenden Gratulatione1l, die nicht zur Tuberkulose gehören, wurde auch noch von anderen Pathologen, so Engel, Gendrin, Robin und besonders von Empis aufgenommen, der für diese Krankheit, um ihre Trennung von der Tuberkulose recht deutlich $z$ machen, den neuen Namen Granulie vorschlug. Obgleich gar kein Zweifel darüber bestehen konnte, daß, wie V irch o w sofort nachwies, diese miliaren Granu lationen nichts anderes als Tuberkel, daB die Granulie nichts anderes als eille Form der Tuberkulose war, so hat diese lrrlehre doch bis in die neueste Zeit hinein Vertreter (Talma, Aufrecht, Behring) gefunden. Es handelt sich hier zunäclst um eine rein pathologisch-anatomische Frage, bei der nur mitzureden beanspruchen kalln, wer genügende eigene makroskopische und mikroskopische Erfahrulgen besitzt, es lohıt sich daher nicht, da diese Voraussetzungen vielfach fehlen, auf diese Streitfrage hier näher einzugehen, zumal atich die experimentellen und bakteriologischen Forschungen allen

1) Virch. Arch. 1861,21 , S. 5.
Zweifelı ein Ende gemacht haben. Wer sich für Einzelheiten interessiert, findet in meiner Abhandlung ,Z Zul Frage nach den Beziehungen der sog. akuten Miliartuberkulose und der Tuberkulose überhaupt zur Lungenschwilddsucht"“, B. kl. W. $1881 \mathrm{Nr}$. 42, sowie in nieinem Beitrage zur Rindfleisch-Festschrift, „Altes und Neues über Lungentuberkulose', 1906, weitere Austührullgen.

Eill scharfer Gegner erwuchs dieser Dualitätslehre in La ën nec, dessen Lehren von dem größten Einfluß auf die Entwicklung der Tuberkuloselehre gewesen sind. Für ihn unterschicden sich die tlicht käsigen Grantulationen von den velkästen wie die grüne von der reifen Frucht; sie gehörten untrennbar zusammen, vor allern auch in den Lungen: es gab für Lä̈nnec auch anatomisch nur eine einheitliche Lungentuberkulose. Lebhaft wurde darüber gestritten, in Frankreich so gut wie in allen anderen Ländern, ob die Lungenveränderungen entzündlicher Natur seien oder nicht, ob es sich um cine Neubildung geschwulstartiger Natur handle, um eine Ausscliwitzung, etwa gar einer vorgebildeten Materie aus dem Blut, welcher Art diese Ausschwitzung sei usw. Vor allem standen sich zwei Lager gegenüber mit dem Feldgeschrei: hie Entzündung, liie Neubildung; wie sehr es sich aber dabei vielfach nur um eillen Streit um Worte gehandelt hat, elgibt sich deutlich aus der Erklärung Julius Vogels, der die Tuberkel für eine bösartige heterologe Neubildung, ein Pseudoplasma, erklärte, das aus eillem ursprünglich flüssigen Exsudat hervorgegangen sei. Für La ënnec beruhte jede Lungenphth se auf Bildung von Tuberkelı, welche niemals entzündlicher Natur seien, sondern Neubildungen wie der Krebs; sie träten sowohl als isolierte Tuberkel auf, als auch in Fornı tuberkulöser lnfiltrationen, aber stets seien die Bildungen aufangs grau, hart, mehr oder welliger durchsichtig, nur bei den Infiltrationen manchmal auch gallertig (das erste Stadium unserer käsigen Pneumonie!), dann gelb und opak, endlich käsig. Sowolıl die aus isolierten (Miliar-) Tuberkeln als auch die aus Tuberkelinfiltrationen hervorgegangene Käsemasse könne erweichen.

Es war ein verhängnisvoller lrrtum La ën necs, der begeisterte Anhänger wie heftige Gegner fand, daß er den verkästen Tuberkel für die reife Frucht hielt, daß er demnach nicht das Knötchen, sondern die käsige Materie für das Kennzeichnende der Tuberkulose erklärte, es war ein weiterer folgenschwerer lrrtum, daß er ohne weiteres aunahm, jede käsige Masse müsse aus der grünen Frucht, der grauen Wucherung, hervorgegangen sein, gleichgültig, wo sie sich finde, ob in den Lungen, ob in skrofulösen Lymphdrüsen, ob in bösartigen Geschwülsten oder sonstwo. Wo Käse war, da war für ihn und scine Anhäıger auch Tuberkulose - und damit waren die Grenzen dieser spezifischen Krankheit aufs ungebührlichste erweitert.

Hier setzte Virchow, auf den Schultern von Vorgängern stehend, ein und wies nach, daß nicht die Käsebildung die Reifung der Frucht bedeute, daß sie nichts für die Tuberkel allein Ken11zeichnendes sei, sondern daß es sich um eine rückgängige Metamorphose, um eine Form des Absterbens handle, die an keinerlei besondere Grundlage gebunden sei. Lebert hatte geglaubt - und viele andere mit ihm -, daß er durch seine Tuberkelkörperchen, die er in jedem Käse auffand, den mikroskopischen Nachweis der Identität allel kësigen Materie geliefert habe, Virchow zeigte aber, daß das Tuberkelkörperchen nichts anderes ist als ein gänzlich verändertes, geschrumpites, zum Teil fettig degeneriertes, atrophisches oder ganz und gar abgestorbenes Giebilde, durch das nur das eine bewiesen wild, daß der käsige Zerfall aus verschiedenen Elementen gleichartige Zersetzungsstoffe fördert. Virchow hatte anfänglich, dem allgemeinen Sprachgebrauch folgend, bei der Verkäsung auch noch von Tuberkulisation gesprochen, gab aber, sobald er das U11zutreffende dieser Bezeichnung erkannt hatte, sie vollkommen auf und ersetzte sie dulch die rein beschreibende Bezeichnung Verkäsung und sprach, wie schon vor ilım Vetter, weder von tuberkulöser noch von skrofulöser, sondern llur mehr von käsiger Materie, weil ebe!1 nur ein Teil dieser Materie der Skrofulose oder Tuberkulose zugehörte.

Hatte Vilchow hierdurch die ulgebührliche Erweitertng des Gebietes der Tuberkulose beseitigt, so schränkte er sie selbst in ungebührlicher Weise ein, indem er nur diejenigen Veränderungen zur Tubelkulose rechnen wollte, welche aus Tuberkeln hervorgegangen waren. Dadurch gelangte er cur Abtrennung käsig entzündlicher, meist als skrofulös bezeichneter Veränderuligen von den tuberkulösen an verschiedenen Orgautn, vor allem an den Knochen (Pottscher Buckel), an den Lymplidrüsen und an den Lungen.

Daraus geht hervor, daß Virchow iın Gegensatze zu Lä̈nnec, der Skrofulose und Tuberkulose identifizierte, zwischen Skrofulose und Tuberkulose einen Unterschied machte. Wenn man Virchows Aeußerungen über diesen Punkt aufmerksam liest, so erhält man den Eindruck, dal zwei Seelen in seiner Brust warell, eine, welche zur Vereinigung von Skrofulose und Tuberkulose hinneigte - hat cr doch immer die Möglichkeit aufrecht erhaltell, die Tuberkulose gleichsam als eine heteroplastische oder metastatische Skrofelbildung zu betrachten - und eiıe andere, welche sich gegen diese sträubte. Die letzte siegte, sodaB Virchow schließlich einen Unterschied machte, indem er unter Skrofulose eine Erkrankung verstand, welcher eine geringere Widerstandsfähigkeit der Gewebe gegen Störungen (Vulnerabilität) der Teile, und geringere Ausgleichsfähigkeit der letzteren (größere Pertinazität der Störungen) zugrundeliege und die an den erkrankten Geweben irritative Veränderungen erzeuge, welche teils den hyperplastischen, teils den entzündlichen Charakter an sich 
trügell. In letzter Beziehung sprach Virchow von einer skrofulösen Entzïndung, während er eine tuberkulöse Entzündung nur in dem Sinne anerkannte, da $\beta$ neben den gewöhnlichen Entzündungs. produkten (wie bei der Meningitis tuberculosa) wirkliche Tuberkel vorhanden sind. Er charakterisierte selbst den Unterschied zwischen beiden mit den Worten, daß die skrofulöse Entzündını eine ätiologische, die tuberkulöse vielmehr eine anatomische Bedeutung habe.

In dieser Unterscheidung liegt meines Erachtens der Schlüsșel zıım Verständnis der Virchow schen Dualitätslehre in bezug auf die käsigen Knochen-, Lymphdrüsen- 1nd vor allem Lungenerkrankungen, denıı nicht n11r die tuberkulöse Entzündıng in dem eben angegebenen Sinne, sondern anch die Tuberkulose überhaupt ist für Virchow ein anatomischer Begriff; hätte er anch sie, wie die skrofulöse Entzündung, vom ätiologischelı Standpunkte aus betrachtet, so wäre er nicht bei einer Scheidung der tuberkulösen von den käsig entzündlichen Veränderıngen geblieben, denn beide haben die gleiche Ursache, das Virus tuberculosum (das Tuberkelgift von Klebs) bzw. den Kochschen Tuberkelbazillus.

Das schließt aber natürlich nicht aus, daß neben der ätiologischen Einheit eine anatomische Verschiedenheit besteht, und diese scharf betont und hervorgehoben zit haben, das ist eben das große Verdienst von Virchow.

$\mathrm{Da} \beta$ in der phthisischen Lunge entzündliche Veränderungen vorkommen, bei denen die Lungenalveolen in mehr oder weniger großen Gewebsabschnitten mit Exsudat gefüllt sind, war schon früheren Untersuchern nicht entgangen; auch an solchen Angaben, daB die Tuberkel mit Entzündung nichts zil tun hätten, also von diesen pneumonischen Veränderungen verschieden seien, hat es nicht gefehlt, aber scharf war die Unterscheidung noch nicht durchgeführt, vor allem war der Anteil aller dieser käsig-entzündlichen Veränderıngen an den Zerstörungen des Lungengewebes bei der Lungenschwiudsucht noch nicht sicher festgestellt, vielmehr behaupteten gerade von ihnen La ënnec und seine Anhänger, daß sie gar keine Rolle spielten, sondern daß einzig und allein die aus einer ursprünglich grauen Wucherung (Nenllilldung) bestehenden tuberku'ösen Veränderungen die Grundlage der Lungenschwindsucht seien. Demgegenüber zeigte Virchow, daß unter der falschen Vorstellung, da $B$ Käsebildung das Charakteristikum der Tuberkulose sei, für verkäste tuberkulöse Neubildung gehalten wırde, was vielfach $1111 \mathrm{r}$ verkästes entzündliches Exsudat war. Er rechnete hierher vor allem die tuberkulöse Infiltration Laënnecs, aber auch vieles, was als isolierte Tuberkel betrachtet worden war, da die käsige Entzündung auch in kleinsten Herdeı (als miliare Pneumonie) auftreten kann, wodırch verkäste Miliartıberkel vorgetäuscht werden konnten und offenbar auch vorgetäuscht worden waren. Es gab jedoch auch noch andere Täuschungsnöglichkeiten dadurch, daß die exsudativ käsige Entzündung nicht auf das alveoläre Lungenparenchym sich beschränkt, sondern auch als Brouchitis bzw. Bronchiolitis caseosa vorkommt: mit käsiger Exsudatmasse ausgefüllte kleinste Bronchien geben aber genau das Bild, das als eingekapselter Tuberkel beschrieben worden ist.

Auch bei der käsigen Pneunonie und Bronchitis stellte Virchow die zellularpathologische Anschauung in den Vordergrund, indem er, alle Ansichten von ungeformten, faserstoffigen, albuminösen, gallertigen Ausscheidungen zurückweisend, erklärte, die käsige Entzündung entwickle sich ganz nach der Art einer entzündlichen Hepatisation dırch zunehmende Anhäıfung von zelligen, zuweilen zellig-fibrinösen Teilen im Lumen der Lungenbläschen und Bronchien, und der Unterschied gegen die gewöhnliche Hepatisation bestehe nur in der frühzeitigenl Verdichtung und Nekrobiose. Dies letzte war freilich nicht wörtlich zı nehmen, denn die späteren Untersuchungen, zunächst von Buhl, haben gezeigt, daß nicht, wie bei der genuinen Pneumonie, gelapptkernige Leukozyten, sondern rundkernige große Zellen als charakteristischer Bestandteil vorhanden sind, die zwar nicht, wie Buhl und andere meinten, desquamierte Epithelzellen, sondern der Hanptsache nach lymphozytäre Elemente sind, sodaß nicht eine Desquamativpneumonie, sondern, im Gegensatz zu der genuinen Pneumonie mit leukozytärem, eine Entzündung mit Iymphozytärem Exsudat auch in der Lunge vorliegt, wie sie in ganz gleicher Weise die Kliniker haben das auch am I.ebenden nachgewiesen, wie die Pathologischen Anatomen an der Leiche - auch an der weichen Hirnhaut, an der Pleura usw. vorkommt. (Wegen zahlreicher Einzelheiten verweise ich auf meine früheren Besprechungen dieses Gegenstandes, von denen ich nur meine Abhandlung über käsige Pneudie Abhandlung Zur Histologie und Aetiologie der Lungenschwindsucht" "in den Nachrichten der Gesellschaft d. Wiss. in Göttingen, Math.-phys. KI., 1901, Heft 2, meinen Bericht ,Welche morphologischen Veränderungen können durch Tuberkelbazillen erzeugt werden?" in den Verhandlungen der D. Pathol. Ges. IV, 1902, die Abhandlung "Altes und Neues über Lutngentuberkulose" in der Rindfleisch-Festschrift 1906 erwähne.)

Den zellularpathologischen Standpunkt kehrte Virchow auch in der Definition des Tuberkels scharf hervor; nicht das Stadium der Verkäsung ist dabei in Betracht zu ziehen, sondern das Stadium incrementi et acmes ins Auge zu fassen, und demnach ist der wahre Tuberkel organisiert in dem modemen Sinne des Wortes: er hat eine zellige Zusammensetzung. Diese Zellen entstammen nach Virchows Meinung wesentlich dem Bindegewebe: indem die Bindegewebszellen in Wucherung geraten, erzeugen sie in regelmäBiger Erbfolge die Zellen der Tuberkel. Demnach können die Tuberkel der Lungen nicht innerhalb der Alveolarlumina gelegen sein, son- dern müssen im Gerüst ihren Sitz haben. Henle hatte zwar schon vor Virch ow s Auftreten in seiner "Rationellen Pathologie" (1847) die schon damals bestehende Kontroverse, ob die Anhäufung der tuberkulöseı oder irgendeiner anderen Materie in dem Lumen der Lungbläschen oder in deren Interstitien statthabe, für müßig erklärt, da von einem interstitiellen Gewebe zwischen den Wänden der Lungenzellen oder gar von einer auf dasselbe beschränkten Exsıdation nicht die Rede sein könne; für ihn bildeten die Tuberkel eine Ablagerung von unvollkommen geronnenem Faserstoff in die natürlichen Hohlräume der Lunge, aber seine Lningentuberkeln waren eben nicht die richtigen tuberkulösen Wuchernngen Virchows und $\mathrm{L}$ a ën $1 \mathrm{ecs}$, sondern waren nichts anderes aIs Virchows käsige Pneumonie, deren Exsudat natürlich nur in die Alveolarhöhlen und nicht zwischen die Alveolarwandungen zu verlegen war, welche letzten zwar nicht Exsudat aufzunehmen, aber als zellenhaltiges Geweb durchaus imstande sind, einer zelligen Wucherung zum Ausgangspunkt zu dienen. Die Verdickung des Lungengerüstes bei der Tuberkelbildung hat Cornil - ich fïhre absichtlich einen Landsmann und Verteidiger La ënnecs an - schon 1867 in seinem mit Hérard herausgegebenen Buche „De la phtisie pulmonaire“" wiederholt gu abgebildet, und, wie das tuberkulöse Grantulationsgewebe, das Epithe vor sich herstülpend, also immer noch außerhalb des Alveolarlumens bleibend, aus dem Lungenorëüst hervorwächst, das hat wieder Cornil in seinem mit Ranvier im Jahre 1869 veröffentlichten Handbuch der pathologischen Histologie in klarster Weise abgebildet, wie denn überhaupr Cornil nicht nitr in diesem Lehrbuch, sondern auch in anderen Veröffentlichungen sich tatsächlich in morphologischer Beziehung durchaus auf den Virchowschen Standpunkt der Verschiedenheit der anatomischen Veränderungen in phthisischen Lungen gestellt hat. Dab trotzdem Cornil in jeder, nicht nur in ätiologischer Beziehung als Verteidiger der La ë nn e cschen Unitäts-,., gegenübe der Virchowschen Dilalitätslehre betrachtet werden wollte, hat offensichtlich nicht wissenschaftliche, sondern andere Gründe.

Man mag über die von Virchow für seine Histiogenese der Tuberkelzellen gelieferten Beweise skeptisch urteilen, die Richtigkeit seiner Annahme ist aber nicht zu bezweifeln, nachdem Baum. garten den sicheren histologischen Nachweis für Teilung und Wucherung. örtlicher Gewebszellen in Gestalt von Karyomitosen geliefert hat. Als Gegengrund kann nicht das sicher festgestellte Vorkommen von anatomischen Tuberkeln in fibrinösem Exsudat oder in Blutgerinnseln angeführt werden, da bekanntlich auch junge Bindegewebszellen wandern können und das Eindringen junger Bindegewebszellen in fibrinöse Massen bei Organisation von Thromben, von Exsindatfibrin usw. mit Leichtigkeit festzustellen ist

Nun hat zwar Virchow die so entstandenen Tuberkel den lymphatigen Geschwülsten oder Lymphomen zugerechnet, abe er hat stets ihre nahe Verwandtschaft mit Granulationsgewebe und Granulomen betont und ja auch eine Erkrankung; deren auch in anatomischem Sinne tuberkulöse Natur ihm freilich noch entgangen war den Lupus, direkt den Granulationsgeschwülsten eingeordnet. Dami stimmt durchaus die Tatsache überein, daß Virchow erklärte, der Tuberkel sähe anfangs wie eine Granulationsbildıng aus, und daß es ihnı sehr wohl bekannt war, da $B$ nicht nur den Lymphdrüsenzellen gleichende Zellen in Tuberkeln vorkommen, sondern auch größere, feinkörnige, großkernige Zellen von mehr epithelioidem Habitıs, in bezug auf welche er beim Lupus ausdrücklich angab, daß sie mi den Epithelzellen des Rete gar nichts zu tun hätten. Endlich kannte Virchow auch die vielkernigen Riesenzellen, die er in tuberkulösen Lymphdrüsen ,in so ausgezeichneter Weise fand, daß man an sogenannte Myeloidgeschwülste denken könnte". Hier stellte er also die Tuberkelriesenzelle neben die Knochenmarksriesenzellen (Myeloplaxeıl, Ostoklasten); daß er aber auch die später so genannte Langhanssche Riesenzelle schon kannte, geht al1s seiner Beschreibung der Riesenzellen in Perlsıchtknoten hervor: vielkernige Elemente, welche schließlich zu den größten Riesenzellen anwachsen können (bis 50 und 60 Kerne), gewölnlich zackig; mit kleinen Stacheln oder langen, oft weiter verästelten Fortsätzen; anßen eine ziemlich derbe Membran, darunter eine feinkörnige Inhaltsmasse, in deren Rinde die Kerne eine Art von Scheide um das homogene Innere bilden. Wenn diese Beschreibung sich auch auf die von Virchow aus unzureichenden Gründen nicht als Tuberkulose anerkamnte Perlsucht des Rindviehes bezog, so wissen wir doch heute, daß er damit die typische Tuberkelriesenzelle vom sogenannten Langhanstypus z11erst beschrieben hat.

Daß Virchow die von anderer Seite längst anerkannte tuberkulöse Natur der Perlsucht nicht anerkannte und nicht erkannte, lag àn seinem starren Festhalten an dem anatomischen Standpunkt, wie er ihn durch seine Untersuchungen am Menschen gewonnen hatte und den er auf die Tiere übertrıg, ohıe zu berïcksichtigen, daß die gleichen Krankheiten bei Tier und Mensch weder physiologisch noch morphologisch völlig gleiche Erscheintungen machen können, da eben Körpertätigkeit und Körperbau verschieden sind (vgl. meine Abhandlung ,Ueber Fütterungstuberkiılose“ in Virch. Arch. 1879, 76). So kam es, daB Virchow in seiner Onkologie erklären konnte, er könne nach eigener Erfahrung nicht sagen, daB er jemals die eigentlichen Tuberkel bei Tieren gesehen hätte, und so erklärt es sich, daß er weiter sagen konnte, noch niemand habe bis jetzt experimentel Tuberkel machen köntlen, was freilich zu einer Zeit geschah, wo erst dürftige Versuche vorlagen, deren Resultate in ihrer Bedeutung noch sehr aggezweifelt wurden (vol. meinen Vortrag in der Medizinischen Gesellschaft „Ueber Tuberkulose“, B. kl. W. 1875 Nr. 12). Aber 
aucl in späterer Zeit, als die beweisendsten experimentellen und experimentell-bakteriologischen Forsclungen schon vorlagen, konnte sich Virchow nicht entschließen, den rein anatomischen gegenüber dem experimentellen Standpunkt aufzugeben. Er trat damit zu sich selbst in Widerspruch, wie ich schon bei früherer Gelegenheit in dieser Wochenschrift dargeleot habe (,R. Virchow und die Bakteriologic", D. m. W. 1910 Nr. 42), da er selbst schon vor langer Zeit erklärt hatte, bei der Beurteiluıg der Infektionskrankheiten erscheine uicht der morphologische $\mathrm{Weg} \cdot$ als der vorzïglichere, ïber die lufektion entscheide allein das Experiment, dem wir uns unterzuordnen hätten.

War denn in Virchows Augen die Tuberkulose eine Infektionskrankheit? Daß etwas Iıfektiöses bei ihr eine Rolle spiele, inısbesondere bei der sekundären sowohl örtlichen wie allgemeinen (hämatogenen) sogenanuten Miliartuberkulose, war ihm sehr wohl bekanut, deun er hat z. B. vom tuberkulösen Geschwür geschrieben, die fortschreitende Eruption neuer Knötchen neben und unter dem Geschwür sei ein unverkeunbares Anzeichen des infektiösen Charakters, und von den geschlossenen tuberkulösen Herden in den Organen, es beginne datın (uach ihrer Erweichung) die infektiöse Sekundäreruption. Virchow kannite die Metastasierung auf dem Lymphweg, er kannte die auf dem Blutwege und erklärte ganz direkt, er glaube an zwei Dingen festhalten zu müssen, zunächst an der Infektionsfïhigkeit der Tuberkel, und zwar nicht bloß iı ihrem käsigen und erweichten, sondern in ihrem Wucherungsstadium, welche nicht nur das Wachstum der Körner zu Nestern und Konglomeratknoten, sondern auch die Dissemination in die Nachbarschaft und die endliche Generalisation auf dem Wege der Metastase erkläre. Was insbesondere die akute allgemeine Miliartuberkulose, auf deren epidemisches Vorkommell Virchow die Aufmerksamkeit lenkte, betrifft, so erklärte er ihr Vorkommeı ohne Käseherd für überaus selteı, erkannte aber trotzdenı einzelne sehr seltene Fälle an, wo diese Primärknoten und Geschwüre ganz fehlen und wo die Miliartuberkulose als Primäraffekt erscheillt.

Hier trat für Virchow eine andere wichtige Grundlage für die Tuberkulose in den Vordergrund, nämlich eiıe spezifische Prädisposition der Gewebe, mochte sie vererbt oder erworben sein. Auch hierbei betonte Virch ow scharf seine zellularpathologische Anschaunng, indem er von der Vererbung sagte, sie sei nicht vom Standpunkte der Humoral-, sondern nur von dem der Zellularpathologie aus zu erklären: die Gewebe seien die Träger der Disposition, die allein, nicht die Krankheit, vererbt, aber auch z. B. durch Trauma erworben werden könne, und bei der einer lokalen Vulnerabilität und ihrem Gegenteil, einer Immunität der Gewebe die allgemeine Vulnerabilität und Inımunität der Individuen gegenüberstehe.

Neuerdings ist es wieder einmal Mode gewordent, jede Prä. disposition z.1 leugnen; frühel gesclıah dies vom ätiologischen, insbesoudere bakteriologischen Staudpunkte aus, jetzt geschieht es vom Standpunkte der Immunitätslehre. Eines ist so einseitig wie das anderc, und das letzte um so uuberechtigter, als es, wie die pathologisch-anatomische Erfahrung schon lange gelehrt hat, eine nennenswerte Immunisierung gegen Tuberkulose überhaupt nicht gibt. Die Bedeutung der örtlichen, erworbenen Disposition tritt insbesondere bei der durch die neuere Unfallmediziı immer bekanıter gewordenen traumatischen! Tuberkulose hervor, die auch schon Virchow nicht unbekannt war (vgl. meineu Vortrag „Ueber Traumen und Tuberkulose der Kuochen und Gelenke" "in den Sitzungsber. d. Preuß. Akad. d. Wiss. 1921 S. 31). Eine andere Frage ist es, ob Virchow die örtliche Disposition nicht überschätzt hat, wenn er meint, sie erkläre die Eruption primär multipler Tuberkel, welche nicht durch Dissemination und Generalisation, sondern auf örtlich e Reize gewöhnlicher Art geschehe. Von einer spezifischeı Substauz im Blute, die der aktive Grund der Tuberkulose sei, wollte Virchow nichts wissen, und er blieb bei dieser Anschauung auch, nachdem das Virus tuberculosum greifbare Gestalt angenommen hatte, tıachdem der Tuberkelbazillus entdeckt war, desséı Beziehungen zur Tuberkulose er nicht leugnete, dessen Nachweis er aber nicht für genügend hielt, um der Tuberkulose den Charakter als Infektionskrankheit, was nicht gleichbedeutend sei mit parasitärer Krankheit, zu verleihen. Der Nachweis von Tuberkelbazillen bei der Perlsucht söhnte ihı anscheinend mit der Vorstellung, daß diese gleich Tuberkulose sei, aus, aber als Koch 1901 in London die völlige Verschiedenlieit der menschlichen Tuberkulose und der Perlsucht des Rindviehes proklamierte, da triumphierte Virchow und wies iu seinenı letzten Vortıage in der Medizinischen Gesellschaft „Ueber Menschen- und Rindertuberkulose" darauf hill, daß er ja die Verschiedenlheit immer bchauptet habe. Abgesehen davon, daß sich späterhiı dic scharfe Kochsche Trennung der Menschen- und Rindertuberkulose nicht hat aufrechterhalten Iassen, hat doch Virchow schon selbst durch die Bezeichnung Rindertuberkulose anerkanıt, da 3 es sich bei ihr nicht um ein Lymphosarkom handelt, und wenn man streng logisch urteilen will, so war doch auch für Koch die Perlsucht iutr diejenige Krankheit des Rindviehes, welche der Tuberkulose beim Meuschen entsprach.

Virchow machte einen Unterschied zwischen bazillärelı, falschen und wirklichen pathologisch-anatomischen Tuberkelı, ohne aber den Unlerschied zwischen beiden genauer auzugeben, und blieb in bezug auf die Nomenklatur ganz auf seinem alten Standpunkt stehen: "man muß die wirklichen pathologischen Tuberkel und nicht die bloß bakteriologischen in den Vordergrund stellen und darf nichts eine Tuberkulose nennen, wobei nicht Tuberkel in derjenigerf Form ent- stehell, wodurch sie sich pathologisch-anatomisch als wirkliche Tuberkel rweisen".

Damit hat also Virchow trotz aller Erungenschaften der experimentellen Pathologie und der Bakteriologie an seinem starren anatomischell Standpunkt festgehalten und besonders in bezug auf die Lungenschwindsucht die nosologische Einheit, wie sie in erster Linie voll $\mathrm{L}$ a ënnec verteidigt worden war, geleugnet. Das ist sehr zu beklagen, denn auch in morphologischer Beziehung finden sich be Virchow selbst schon Andentungen dafür, daß den Tuberkeln, deu Knötchenbildungen, doch nicht unbedingt die alles beherrschende Stellung zukommt, welche Virchow für sie bis zuletzt in Auspruch nahm. Bei der Beschreibung der Konglomerattuberkel des Gehirns und Rückenmarks heißt es nämlich in der Onkologie, sie zeigten ganz deutlich, daß das Wachstum der Knoten durch Apposition oder Juxtaposition erfolge und daß die Apposition nicht durch primär käsige Schichtelı, sonderı durch Zonen neuer grauer Wucherung, gewöhnlichx) in Form von miliaren Knoten erfolge, welche allerdings sehr frühzeitig zusammenfließeı und dann eine mehr zusammenhängende Infiltration um den Mutterknoten bildeı, in der schon während der eigentlichen Wucherungsepoclac keine getrennten Knötchen mehr wahrnehmbar sind ${ }^{1}$ ). Mit den Nachsatze: ,immerhin ist das, was juxta- oder apponiert wird, wucherudes Gewebe", wird m. E. schon zugegeben, daß es tuberkulöses Gewebe ohne Tuberkel gibt. Damit war also eigentlich schon vori anatomischen Standpunkt aus der Tuberkel entthront, und der Schritt zur Aulerkenuung der aus diffuser Gewebswucherung hervorgeheıden Verkäsung sog. skrofulöser, mit käsiger Entzüıdung begabte: Lymphdrüsell als tuberkulöser, das Virus tuberculosum, die Tuberkelbazillen enthaltender Lymphdrüsen wärc kein großer mehr gewesell. Virch ow hat ihn nicht getan. Ist dadurch seine morphoogische Trennuıg der tuberkulösen, d.h. durch die Entstehung eines besondereı wucherıden Gewebes gekenuzeichneten Veränderungen in phthisischen Lungen, von den entzündlichen, d. h. durch einen besonderen Verlauf (und wie jetzt feststeht, durch besondere zellige Bestandteile) ausgezeichneten exsudativen weniger gerechtfertigt? Ist dadurch dic vou Virchow aufgestellte morphologische Dualität der Veränderungeı in schwindsüchtigen Lungen hiufällig geworden? Weder hierdurch noch durch die Behauptung, daß zwischen den Tuberkelgranulationen und den käsig-pneumonischen und -bronchitischeı Veränderungeı nur graduelle Unterschiede bestünden. Es soll Pathologen geben, welche ihre Schüler lehreı, die Virch ow sche Dualitätslehre der Lungensclıwindsucht sei ein überwuıdener Standpunkt. Wenn man aber einen Blick in die Lehrbücher der Pathoogischen Anatomie tut, so wird man bald eines Besseren belehrt, denn überall findet man ncben den Tuberkeln die käsige Pneumonie beschrieben, und nicht nur in Dentschland, sondern auch in dem Lande, das die Geburtsstätte der gegenteiligen $L$ a ë n n e cschenl Anschauung ist, in Frankreich, wo, wie schon erwähnt, der bis in die nenere Zeit hinein maßgebende Pathologische Anatom Cornil neben anderen Forschern sehr deutlich die exsudativen von den produktiven Vorgängen sondert und auch die lobären Formen der käsigen Pneumonie sehr wohl kaunte. Das Zugeständnis, es sei tatsächlich festgestellt, daf der Bazillus fähig ist, schwindsüchtige Veränderungen $\mathrm{zu}$ erzeugen, welche nicht Tuberkel sind (Il est en effet démontré que la bacille est capable d'engendrer des lésions phtisiogènes qui ne sont pas des tubercules ...) ist völlig beweiskräftig.

Wie steht es nun mit den Klinikerı? F. Klemperer schreibt nicht mit Unrecht, gegenüber La ë nnecs Unitäts- habe Virch ows Dualitätslehre klinisch einen Rückschritt bedeutet, aber der Ausspruch ist nur insoweit richtig, als es sich um die Abgrenzung derjenigen Krankheit handelt, welche - voul den Mischinfektionen abgesehell - der Lungenschwindsucht, der Lungenphthise zugrundieliegt, derjelligen Krankheit, welche wohl auch in alle Zukunft Tuberkulose wird. Welche Verwirring die Proklamation der ätiologischen Dualität der Lungenschwindsucht angerichtet hat, zeigt am schlagendsten die Behauptung Niemeyers, die größte Gefahr für einen Phthisiker sei die, daß er tuberkulös werde. Aber diese Verwirrung war nutr vorübergehend und wurde je länger je mehr durch die Fortschritte der experimentellen Pathologie und der Bakteriologie völlig behobell. Voll iht ist die morphologische Virchow sche Dualitätslehre vöHlig unabhängig; und daß diese erhalten geblieben ist, daß sie
auch auf klinischer Seite sich durchgesetzt hat, zeigt wieder ein Blick In die allgemeine und in die fachliche klinische Literatur, denu
uiberali fiudet man die puleumonisclie Form del Tuberkulose, insbesondere auch die reinen bronchopneumonischefl, die lobulären und lobären käsigen Hepatisationen als eine besonders zu beachtende Art der Lungenveräıderuıgen aufgeführt und eingehend erörtert.

Die jüngst abgehaltene Versammlung der Deutschen Gesellschaft für Innerc Mediziı hat für das Gesagte eine glänzende Bestätigung gebracht. Nicht nurr ist von hervorragender pathologisch-anatomischer Seite (Aschoff) vol1 neuem die Gegenüberstellung einer wesentlich produktiven Norm der Lungenplithise $\mathrm{zu}$ einer wesentlich exsudativea vorgenommen worden, sondern es ist auch von klinischer Seite gezeigt oder mindestens zu zeigen versucht worden, daß man die Unterscheidung der beiden Formen, der produktiven und exsudativen Vorgänge, an der Hand des Röntgenbildes auch klinisch durchführen könne. Es liegt auf der Hand, wie wichtig für die klinische Beurteilung des Falles das Vorherrschen der langsamer fort- 
schreitenden und zerstörenden produktiven Veränderungen oder der schneller zur ausgedehnten Verkäsung und zur Zerstörung des Gewebes führenden exsudativen Vorgänge sein muB, wie dementsprechend auch bei der Therapie hierauf Rücksicht genomnen werden muß, zumal, wenn es sich herausstellen sollte, daß bei den Exsudationen besondere Toxine in Wirksamkeit treten.

Virchow hat also nicht nur das Verdienst, die Käsemasse mitsamt dem Le b e rtschen Tuberkelkörperchen ihrel pathognomonischen Bedeutung entkleidet, die Histologie und Histogenese des miliaren Tuberkels genau festgestellt, die Bedeutung der Disposition klargelegt zll haben, sondern er hat die komplizierten anatomischen Veränderungen bei der Lungenschwindsucht in ihre einzelnen Komponenten zerlegt und damit nicht nur die Pathologische Anatomie der Lungenphthise wesentlich gefördert, sondern auch, nachidem sein Irrturn inl betreff der Aetiologie korrigiert war, für die klinische Diagnose wie für die Prognose und Therapie der Lungentuberkulose eine wertvolle und unverrückbare Grundlage geschaffen. 